\title{
Clinical features and prognosis of breast cancer with gastric metastasis
}

\author{
JOOHYUN HONG ${ }^{1}$, YOUJIN KIM ${ }^{1}$, JANGHO CHO ${ }^{1}$, SUNG WON LIM $^{1}$, SONG EE PARK ${ }^{1}$, \\ HEE KYUNG KIM ${ }^{1}$, HANSANG LEE ${ }^{1}$, SOO YOUN CHO ${ }^{2}$, JI-YEON KIM ${ }^{1}$, \\ JIN SEOK AHN ${ }^{1}$, YOUNG-HYUCK IM ${ }^{1}$ and YEON HEE PARK ${ }^{1}$ \\ ${ }^{1}$ Division of Hematology-Oncology, Department of Medicine; ${ }^{2}$ Department of Pathology, \\ Samsung Medical Center, Sungkyunkwan University School of Medicine, \\ Seoul 06351, Republic of Korea
}

Received February 5, 2018; Accepted October 12, 2018

DOI: $10.3892 / \mathrm{ol} .2018 .9754$

\begin{abstract}
Breast cancer rarely metastasizes to the gastrointestinal tract, including the stomach. Due to the rarity of this metastasis, it is occasionally confused with a primary stomach malignancy. However, discriminating characteristic features with clinical implications may exist. The aim of the current study was to analyze the clinical features and prognosis of breast cancer with gastric metastasis. Between January 1994 and October 2016, 13 patients at Samsung Medical Center (Seoul, Korea) were clinically or pathologically determined to have breast cancer with gastric metastasis. The present study retrospectively collected clinicopathological data from the electronic medical records of these 13 female patients. At breast cancer diagnosis, the median patient age was 45 years. A total of 7 patients $(53.8 \%)$ presented with invasive lobular carcinoma (ILC) and $6(46.2 \%)$ with invasive ductal carcinoma. Of the 13 patients, 11 were stage I-III at initial breast cancer diagnosis and underwent surgery. Positivity of breast cancer tissue samples for estrogen receptor (ER), progesterone receptor (PR) and human epidermal growth factor receptor 2 (HER2) was 92.3, 76.9 and $0 \%$, respectively. Positivity of gastric metastasis lesions, based on immunohistochemistry results, was $81.8,50$ and $0 \%$ for ER, PR and HER2, respectively. The stomach was the location of the first metastatic lesion in 6 out of the 11 patients $(54.5 \%)$ with de novo stage I-III cancer. The median time interval from initial breast cancer diagnosis to stomach metastasis was 77.5 months. The 3 -year survival rate was $79.1 \%$, and the estimated mean survival time was 35.1 months. Breast
\end{abstract}

Correspondence to: Dr Yeon Hee Park, Division of HematologyOncology, Department of Medicine, Samsung Medical Center, Sungkyunkwan University School of Medicine, 81 Irwon Road, Gangnam, Seoul 06351, Republic of Korea

E-mail: yhparkhmo@skku.edu

Key words: breast neoplasms, stomach neoplasms, neoplasm metastasis cancer with gastric metastasis is rare, and due to this fact, a thorough pathological review and greater clinical suspicion are required in these cases.

\section{Introduction}

Breast cancer had one of the highest incidence rates and was a leading cause of cancer-associated mortality worldwide in 2016, and its incidence is continuously increasing (1). Breast cancer is a heterogeneous disease comprising luminal type A, luminal type B, human epidermal growth factor receptor 2 (HER2)-positive and basal-like breast cancer. Clinical features, treatment and prognosis differ depending on the subtype (2). The most common metastatic sites of breast cancer are the bone, brain, liver and lung, however, patterns of metastasis are affected by the breast cancer subtype (3-5). By contrast, breast cancer rarely metastasizes to the gastrointestinal tract, including the stomach, and few studies and case reports concerning gastric metastasis from breast cancer can be found (6-16). Chemokines and their receptors, including CXCR4 and CCR7, are critical for regulating the location of metastasis. Their respective ligands CXCL12/SDF-1 $\alpha$ and CCL21/6Ckine demonstrate peak levels of expression in the primary organs of breast cancer metastasis (17). Low incidence of metastasis to the gastrointestinal tract may be due to low expression of chemokine receptor ligands at this location. Due to its rarity, breast cancer with gastric metastasis is occasionally confused with a primary malignancy in the stomach. However, discriminating characteristic features with clinical implications may exist. Depending on whether it is primary or secondary, the treatment and prognosis are different. The current study aimed to identify the clinical features and prognosis of breast cancer with gastric metastasis. A total of 13 patients with breast cancer and gastric metastasis were analyzed. Invasive lobular carcinoma (ILC) or luminal-type breast cancer tends to metastasize to the stomach at a higher frequency compared with other types of breast cancer. In addition, the stomach was one of the first metastatic lesion locations in the patients in the present study. These patients had a more favorable prognosis compared with patients with primary gastric cancer. 


\section{Materials and methods}

Study population. The present study was a retrospective, single-center study. Between January 1994 and October 2016 in Samsung Medical Center (Seoul, Korea), 21,716 patients with breast cancer underwent esophagogastroduodenoscopy (EGD) prior to or following breast cancer diagnosis. Among them, 66 patients with breast cancer had gastric carcinoma according to the pathology reports from the biopsies. A total of 13 patients (median age, 45 years; range, 38-65 years) with breast cancer presented with gastric metastasis and 47 had double primary cancer. A total of 2 patients had a previous gastric malignancy prior to breast cancer diagnosis. It was not possible to determine whether 4 patients had double primary cancer or breast cancer with gastric metastasis (Fig. 1). Pathology specialists in the hospital determined whether a gastric lesion was primary or secondary.

Out of the 13 patients, 11 were determined to have breast cancer with gastric metastasis according to pathological analysis using immunohistochemistry (IHC) staining with the following biomarkers: Estrogen receptor (ER), progesterone receptor (PR), HER2, gross cystic disease fluid protein 15 (GCDFP-15), cytokeratin 7, GATA-binding protein 3 and E-cadherin. Only 2 patients underwent staining for GATA-3 and 1 patient underwent staining for E-cadherin, therefore, these data are not shown. An additional 2 patients with breast cancer, who had gastric carcinoma at EGD, were also included into this group clinically, as their gastric lesions responded to subsequent breast cancer chemotherapy. The samples obtained from these 2 patients were assessed by EGD without IHC staining.

Data collection. Clinicopathological data from the electronic medical records were collected. The variables considered for the sample collection of the present study were the following: Age, sex, stage according to the 7th edition of the American Joint Committee on Cancer (AJCC) staging system (18), pathology, hormone receptor status of breast cancer, HER2 status, treatment modalities, including surgery, chemotherapy, radiotherapy and hormone therapy for breast cancer, time interval to other metastasis and gastric metastasis, gastric symptoms, endoscopy results according to Borrmann's classification (19), pathology and hormone receptor status of metastasis, other IHC staining markers of gastric metastasis, including GCDFP-15, treatment for gastric metastasis and follow-up data.

IHC staining. Breast and gastric tissue samples were embedded in paraffin and fixed in a $10 \%$ buffered formalin solution for 24-48 h at room temperature. Each sample was cut into 5-mm thick sections. In contrast with the samples of breast cancer, not all samples of gastric metastasis underwent IHC staining with anti-ER (1:200, catalog no. 6F11; Novocastra Laboratories Ltd., Newcastle upon Tyne, UK), anti-PR (1:100; clone 16, Novocastra Laboratories Ltd.), anti-HER2 (pre-diluted; clone 4B5; Ventana Medical Systems, Inc., Tucson, AZ, USA) or anti-GCDFP-15 (pre-diluted; clone EP1582Y; Sigma-Aldrich; Merck KGaA, Darmstadt, Germany) as not all samples were originally considered to be metastasis. A total of 11 out of 13 samples underwent IHC staining for ER. A total of 10, 9 and 10 samples underwent staining for PR, HER2 and GCDFP-15, respectively. Slides were incubated with anti-ER and anti-PR primary antibodies at room temperature for $15 \mathrm{~min}$ or at $37^{\circ} \mathrm{C}$ for $32 \mathrm{~min}$ with anti-HER2 and anti-GCDFP-15 primary antibodies. Subsequently, Bond Polymer Refine Detection kit (Leica Biosystems, Newcastle upon Tyne, UK) was used to detect ER and PR, and ultraView Universal DAB Detection kit (Ventana Medical Systems, Inc.) was used to detect HER2 and GCDFP-15. Stained slides were evaluated using a light microscope (magnification, x100-200). A cut-off value of $\geq 1 \%$ stained tumor nuclei was used to determine samples as ER-positive or PR-positive. Samples were considered as HER 2 positive if samples exhibited IHC $3+$ positive staining or a positive silver in situ hybridization (SISH) result. A diffuse intense circumferential membrane 'chicken-wire' staining pattern in $>10 \%$ of the tumor was scored as IHC $3+$. Tumors with circumferential membrane staining demonstrating a thin pattern of staining and/or heterogeneity in staining distribution in $\leq 10 \%$ of tumor cells were scored as IHC $2+$. Tumors with absent or weak membrane staining were scored as 0 or IHC $1+$ (20). If samples exhibited IHC $2+$ staining, HER2 gene status was evaluated by HER2 SISH. HER2 SISH was considered as positive if the average HER 2/CEP17 ratio was $\geq 2.0$ or the average HER2 copy number was $\geq 6.0$ signals/cell. For GCDFP-15, any cytoplasmic staining was considered as positive.

Statistical analysis. Kaplan-Meier curves were used to measure overall survival rate and time following gastric metastasis, and significant differences were identified by a log-rank test. $\mathrm{P}<0.05$ was considered to indicate a statistically significant difference. Statistical analysis was performed using SPSS software (version 24; IBM Corp., Armonk, NY, USA). Data are presented as the median \pm standard deviation.

Ethical considerations. The present study was approved by the Institutional Review Board of Samsung Medical Center (approval no. 2017-11-097-002). The requirement for informed consent was waived due to the retrospective nature of the study.

\section{Results}

Patient characteristics. Characteristics of the 13 patients with breast cancer are presented in Table I. The median age of these patients at the time of breast cancer diagnosis was 45 years. A total of 7 (53.8\%) patients presented with ILC and 6 (46.2\%) with invasive ductal carcinoma. Positivity in tissue cancer samples was $92.3 \%$ for ER and $76.9 \%$ for PR. All samples were negative for HER2. Out of the 13 tumors, $11(84.6 \%)$ were stage I-III and 2 patients (15.4\%) had stage IV gastric metastasis at initial breast cancer diagnosis based on the 7th edition of the AJCC cancer staging system. All patients with de novo stage I-III breast cancer underwent surgery, consisting of a mastectomy in $9(81.8 \%)$ patients and breast-conserving surgery in $2(18.2 \%)$ patients. 2 patients with stage IV breast cancer received chemotherapy initially instead of surgery.

Characteristics of the gastric metastasis. Characteristics of the gastric metastasis from breast cancer are presented in 


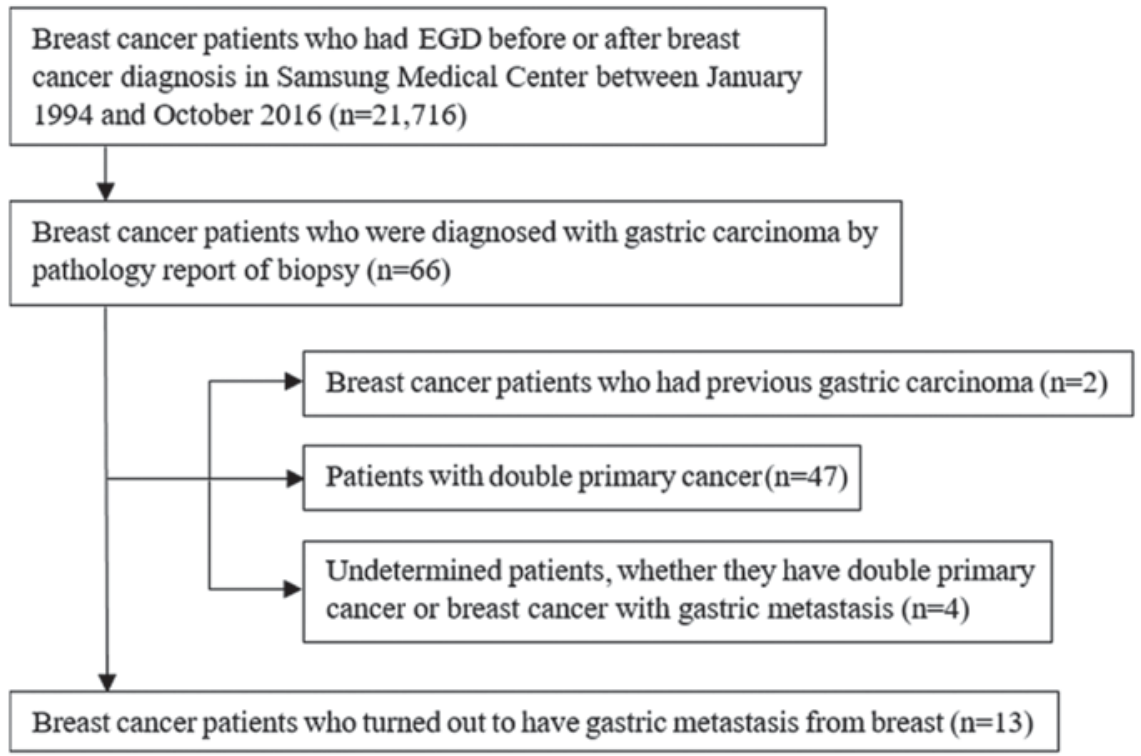

Figure 1. Patient flow chart for collection of data. EGD, esophagogastroduodenoscopy.

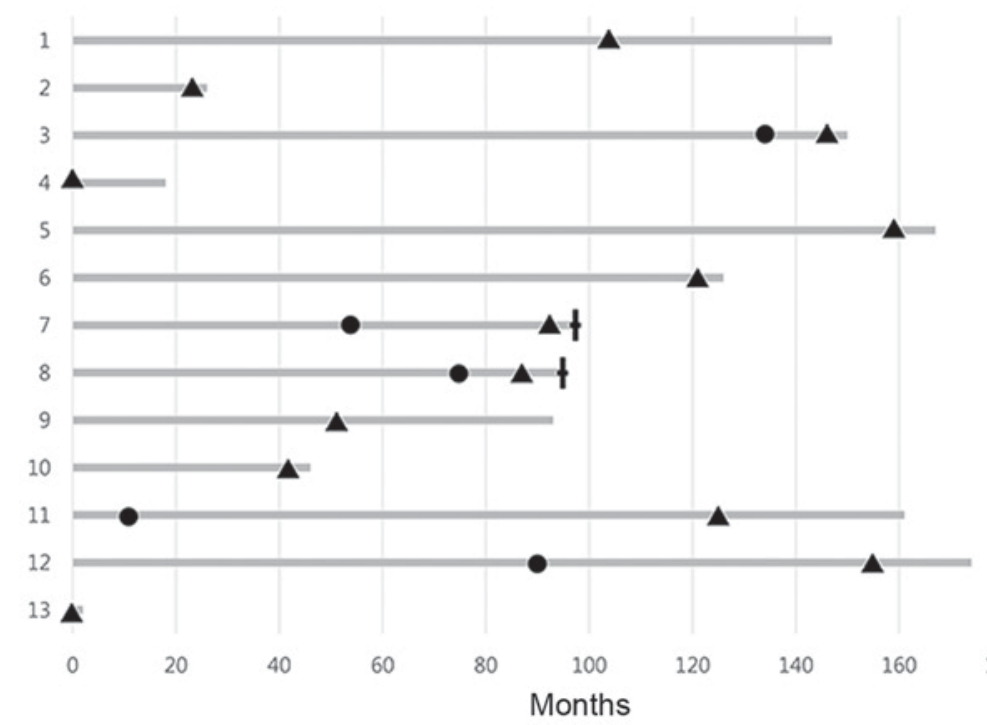

- Time of distant metastasis other than stomach metastasis

$\Delta$ Time of gastric metastasis

† Death

180200

Figure 2. Time interval from breast cancer diagnosis to distant metastasis, and then to gastric metastasis and final follow-up. Each bar represents a patient with breast cancer and gastric metastasis. ${ }^{\bullet \bullet} \mathbf{\Delta}$ Time interval (months) from breast cancer to distant metastasis, and from distant metastasis to gastric metastasis, respectively. The patient succumbed.

Table II. The stomach was the first metastatic lesion location in $6(54.5 \%)$ out of the 11 patients with de novo stage I-III cancer. The median time between breast cancer and gastric metastasis was 104 months for these patients. For the 6 patients where gastric metastasis was the initial metastatic lesion, the median time to gastric metastasis was 77.5 months. For the other 5 patients, the median time between other initial distant metastasis and gastric metastasis was 42 months, where initial metastatic lesion location differed among patients. One of these 5 patients had initial lung and bone metastasis following gastric metastasis, while another patient also had bone metastasis initially, following gastric metastasis. The third patient had liver metastasis initially, followed by gastric metastasis and the fourth patient had bone metastasis initially, followed by metastasis in each ovary and then gastric metastasis. The last of these 5 patients had pancreatic metastasis, followed by metastasis of the ureter and finally gastric metastasis. A time graph for breast cancer and gastric metastasis is presented in Fig. 2. Of the 11 patients for whom details regarding any related symptoms were retrieved by electronic medical records, 7 (63.6\%) had symptoms, including melena, hematemesis, vomiting, poor oral intake, dyspepsia and abdominal pain, while 4 (36.4\%) patients exhibited no symptoms. A total of 7 out of 13 patients underwent EGD due to the aforementioned symptoms, 3 underwent EGD for routine check-ups without symptoms and 1 underwent EGD as gastric malignancy was suspected at follow-up abdominal computed tomography (CT) scan for disease evaluation of chemotherapy. The remaining 2 patients underwent EGD at other hospitals for unknown reasons; therefore information regarding their 
Table I. Characteristics of patients with breast cancer.

\begin{tabular}{lrr}
\hline Variable & Value \\
\hline Median age (range), years & $45(38-65)$ \\
Sex, n (\%) & 0 & $(0.0)$ \\
Male & $13(100.0)$ \\
Female & \\
Pathology, n (\%) & 6 & $(46.2)$ \\
IDC & 7 & $(53.8)$ \\
ILC & \\
Breast cancer receptor, n (\%) & $12 \quad(92.3)$ \\
ER & 10 & $(76.9)$ \\
PR & 0 & $(0.0)$ \\
HER2 & 11 & $(84.6)$ \\
AJCC stage, n (\%) & 2 & $(15.4)$ \\
Stage I-III & & \\
Stage IV & 9 & $(81.8)$ \\
Surgery, n (\%) & 2 & $(18.2)$ \\
Mastectomy & & \\
BCS & & \\
\hline IDC, invasive ductal carcinoma; ILC, invasive lobular carcinoma; & \\
ER, estrogen receptor; PR, progesterone receptor; HER2, human \\
epidermal growth factor receptor 2; AJCC,American Joint Committee \\
on Cancer 7th edition; BCS, breast-conserving surgery. \\
\hline
\end{tabular}

symptoms could not be collected. A total of $9(81.8 \%)$ patients with gastric cancer had tumors that were ER-positive, and 5 out of $10(50 \%)$ had tumors that were PR-positive. Among the nine gastric tumor samples that underwent IHC staining for HER2, all tumors were HER2-negative. Among the 10 patients who underwent GCDFP-15 staining, 6 (60\%) exhibited positive results.

Treatment modalities of the breast cancer. The treatment of the breast cancer, including surgery, adjuvant chemotherapy, adjuvant radiotherapy, hormone therapy, palliative chemotherapy and intrathecal methotrexate is presented in Table III. In addition, Table III also presents the treatment of the gastric metastasis, including palliative chemotherapy, intrathecal methotrexate, radiotherapy, hormone therapy and whole-brain radiotherapy.

Prognosis of gastric metastasis. Kaplan-Meier curves are presented in Fig. 3. The 3-year survival rate was $79.1 \%$, and the estimated mean survival time was 35.1 months. A total of 2 patients succumbed to their disease, and 8 patients remained alive with the disease at follow-up. When comparing 6 patients who had initial gastric metastasis with 5 patients who had subsequent gastric metastasis following initial other distant metastasis, the 3-year survival rate of the former was $100 \%$, while that of the latter was $53.3 \%$. However, there was no significant difference $(\mathrm{P}=0.138)$ between the two, due to the small number of cases. Further extensive follow-up is required to confirm the 5-year survival rate and events.
Table II. Characteristics of patients with gastric metastasis from breast cancer.

\begin{tabular}{ll}
\hline Variable & Value \\
\hline $\begin{array}{l}\text { Median (range) time to gastric metastasis } \\
\text { from breast cancer }(\mathrm{n}=11)^{\mathrm{a}} \text {, months }\end{array}$ & $104(24-159)$ \\
$\begin{array}{l}\text { Median (range) time to gastric metastasis as } \\
\text { initial metastasis from breast cancer }(\mathrm{n}=6)\end{array}$ & $77.5(24-159)$ \\
Median (range) time to gastric metastasis from & $42(12-114)$ \\
another initial distant metastasis $(\mathrm{n}=5)$ & \\
Symptoms, $\mathrm{n}(\%)$ & \\
Yes & $7(63.6)$ \\
No & $4(36.4)$ \\
Stomach cancer receptor, $\mathrm{n}(\%)$ & \\
ER & $9(81.8)$ \\
PR & $5(50.0)$ \\
HER2 & $0(0.0)$ \\
GCDFP-15 & $6(60.0)$ \\
\hline
\end{tabular}

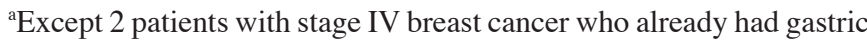
metastasis at initial diagnosis. ER, estrogen receptor; PR, progesterone receptor; HER2, human epidermal growth factor receptor 2; GCDFP-15, gross cystic disease fluid protein 15 .

A patient with gastric metastasis as the initial metastasis. Among the 13 patients with breast cancer and gastric metastasis, only 1 had sole gastric metastasis as the initial metastasis. Even though the stomach lesion was a sole cancer lesion in this patient, it was determined not to be primary, but metastatic, following thorough review and discussion with a pathology specialist. Therefore, rather than surgery or endoscopic treatment, this patient was treated with 2,000 mg capecitabine (Roche Diagnostics, Basel, Switzerland) every 3 weeks for 4 cycles and a reduced dose of 1,500 mg due to diarrhea every 3 weeks for 3 cycles. The last noted response was partial. Another patient had gastric metastasis with regional lymph node metastasis at the initial metastasis (Figs. 4 and 5). Treatment consisted of letrozole (Novartis International AG, Basel, Switzerland), exemestane (Pfizer Inc., New York, NY, USA) plus everolimus (Novartis International), weekly paclitaxel (Hospira; Pfizer, Inc.), doxorubicin (Boryung, Seoul, Korea) plus cyclophosphamide (Baxter Inc., Deerfield, IL, USA), and capecitabine (Roche Diagnostics) until the last follow-up. The remaining patients had other distant metastasis in addition to that of the stomach. These patients did not undergo stomach surgery, but instead received chemotherapy, radiotherapy, hormone therapy or no treatment (Table III).

EGD results of gastric metastases. EGD results of patients with gastric metastasis were diverse in terms of location, size, number, shape, and presence or absence of bleeding, erosion or ulceration (Fig. 6). Fig. 6A presents white discolored mucosal lesion with speculated edge, Fig. $6 \mathrm{~B}$ presents round polypoid lesions with or without ulceration, Fig. $6 \mathrm{C}$ presents diffuse infiltrative lesion with spontaneous bleeding and 


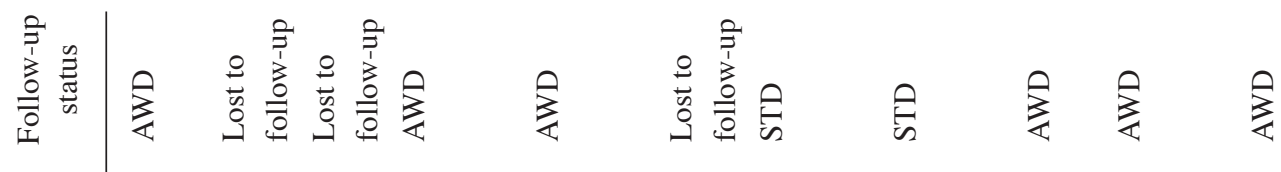

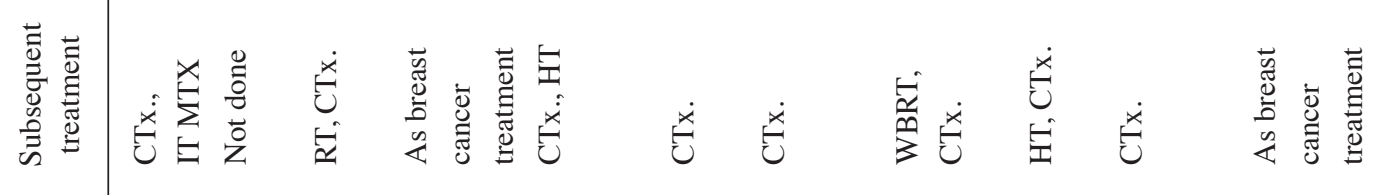

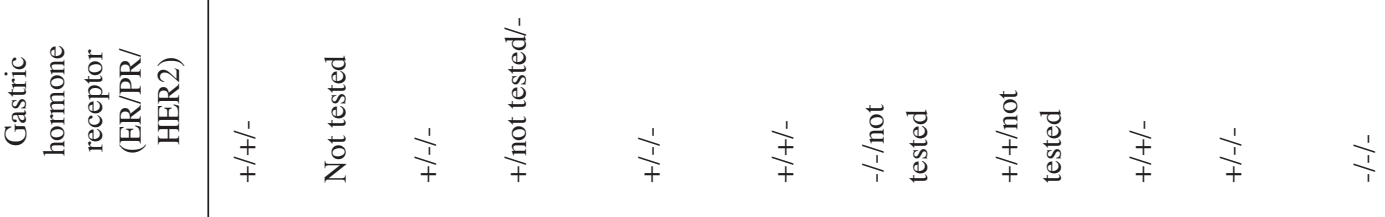

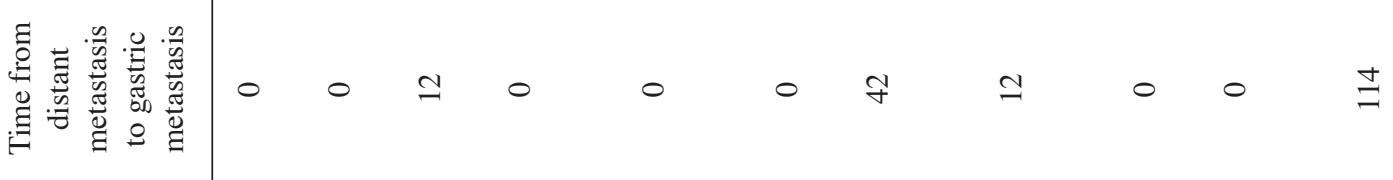

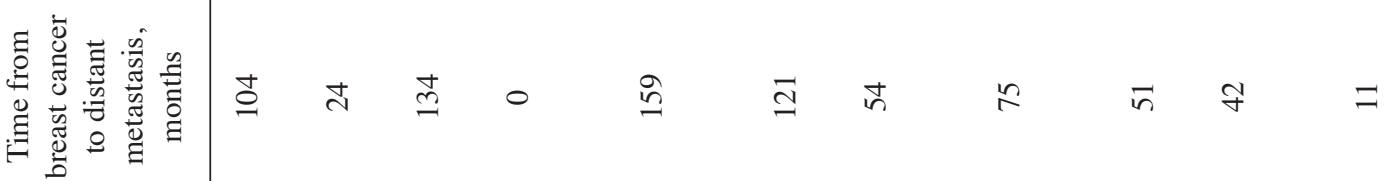

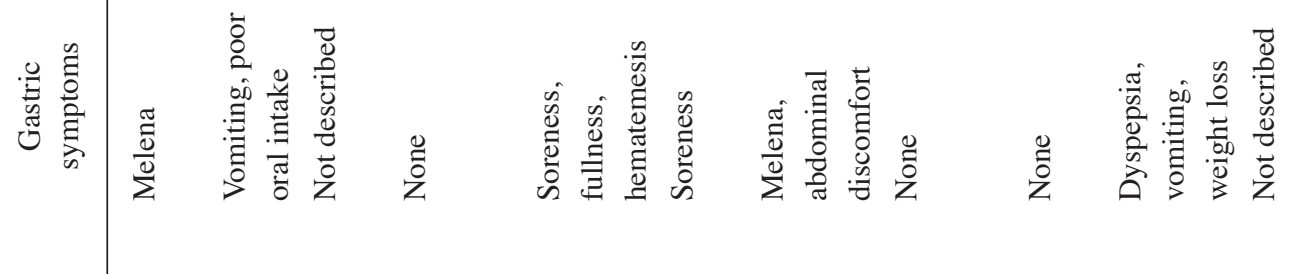

壳

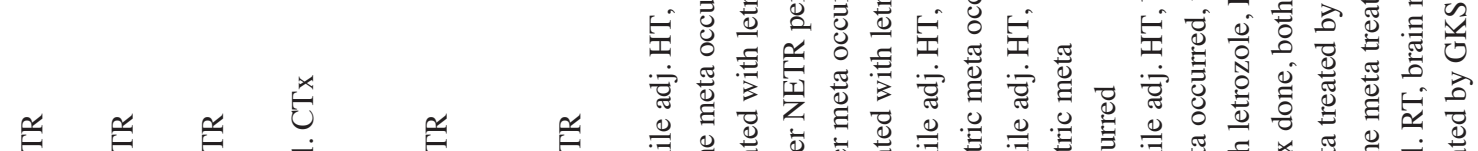

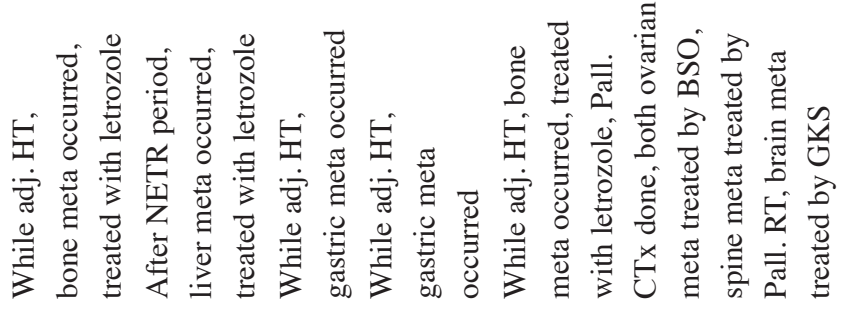

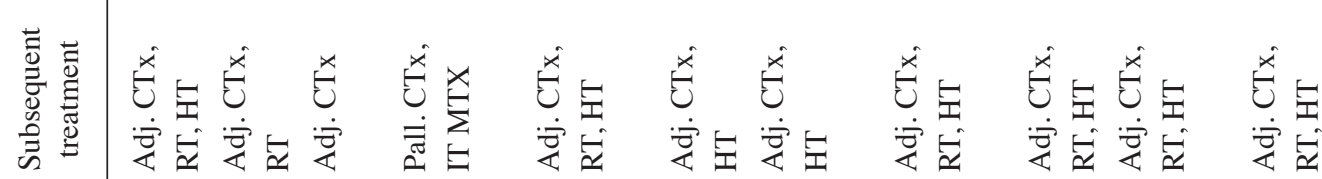

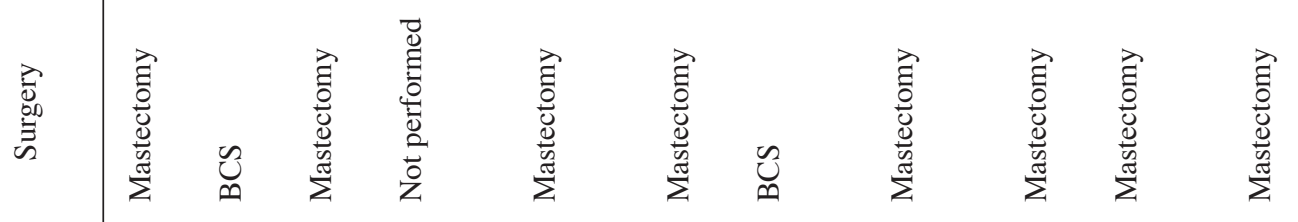

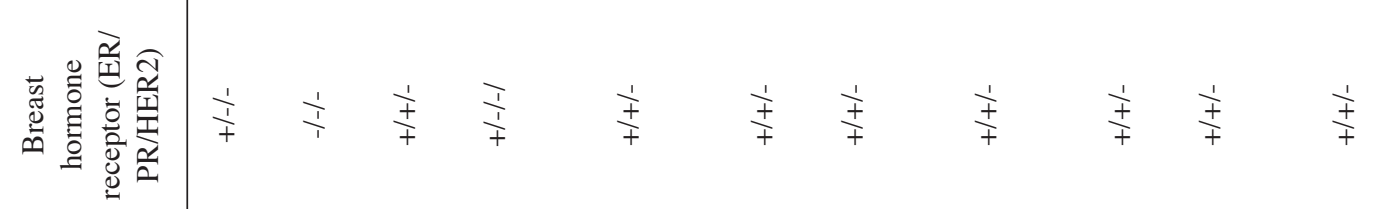
离 


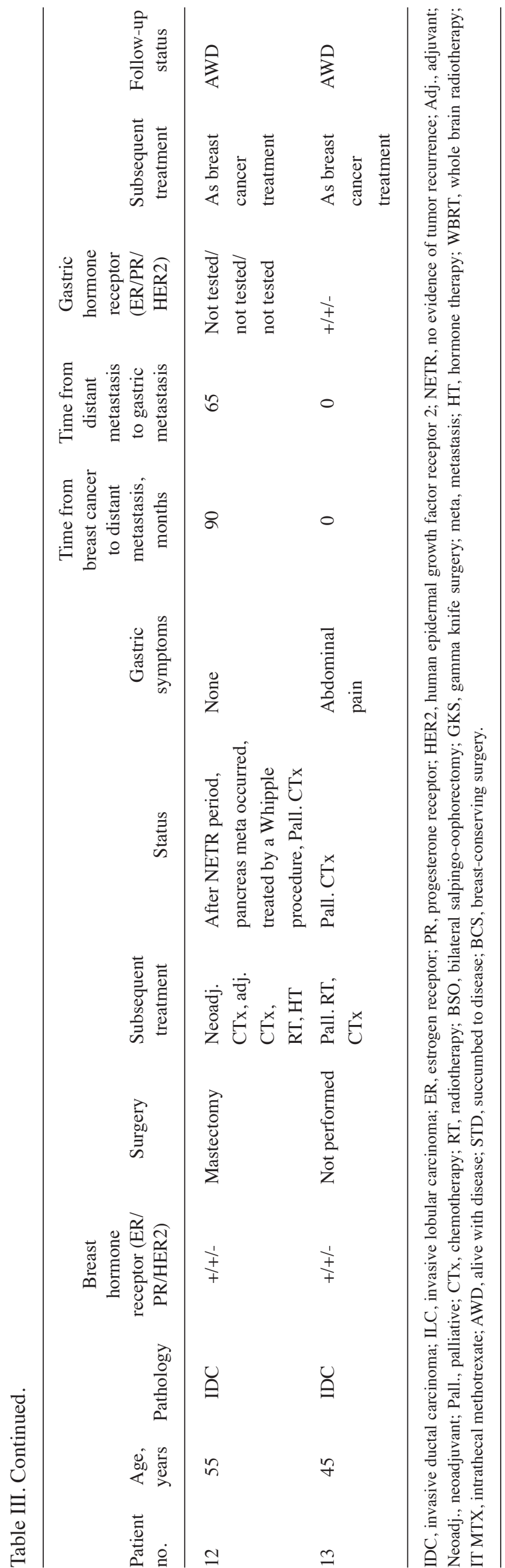

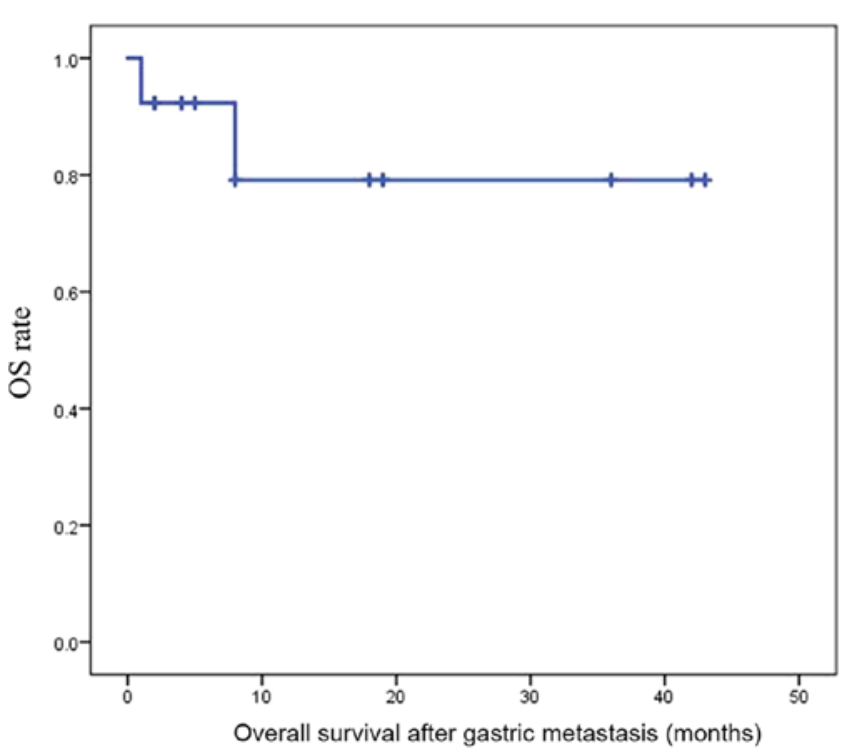

Figure 3. OS rate among patients with breast cancer with gastric metastasis. OS, overall survival.

fold thickening mimicking Bormann type IV, and Fig. 6D presents deep ulcerative lesion with yellowish exudate and spontaneous bleeding. Gastric metastasis can be located anywhere from the fundus to the antrum, even in the lesser curvature and posterior wall side of the remnant stomach. One of the patients underwent a Whipple procedure for pancreatic metastasis prior to gastric metastasis. A gastric lesion was suspected subsequent to a follow-up abdominal CT scan for disease evaluation of chemotherapy. EGD was performed and a gastric lesion in the remnant stomach was found (Fig. 7). Sizes ranged between 0.5 and $10 \mathrm{~cm}$. While 9 patients $(69.2 \%)$ presented with solitary lesions, the other patients (30.8\%) presented with 2 or multiple lesions that increased the suspicion of metastasis. Shapes were diverse, including elevated mucosal lesions or polyps, with or without erosion or ulceration, and infiltrative or ulcerative lesions, which can mimic early gastric cancer to advanced gastric cancer. In terms of bleeding, 1 patient had a mucosal elevated lesion with oozing bleeding, and another patient had an ill-defined nodular lesion with spontaneous bleeding. In addition, 1 patient who presented with melena received hypertonic saline mixed with epinephrine and argon plasma coagulation for spontaneous bleeding. There were no specific EGD results to diagnose gastric metastasis. It is difficult to consider gastric metastasis, particularly in the setting of a solitary gastric lesion. Taking the aforementioned into consideration, clinical suspicion and obtaining a full history of patients in addition to an adequate biopsy of the gastric lesion are crucial.

Undetermined patients. A total of 4 patients had undetermined status, as data were not available. Terminal-stage breast cancer was indicated in 3 patients who were diagnosed with gastric carcinoma as they had gastric symptoms. Since the patients were diagnosed with terminal-stage breast cancer, further evaluation and management was deferred, and they succumbed in the following months. 

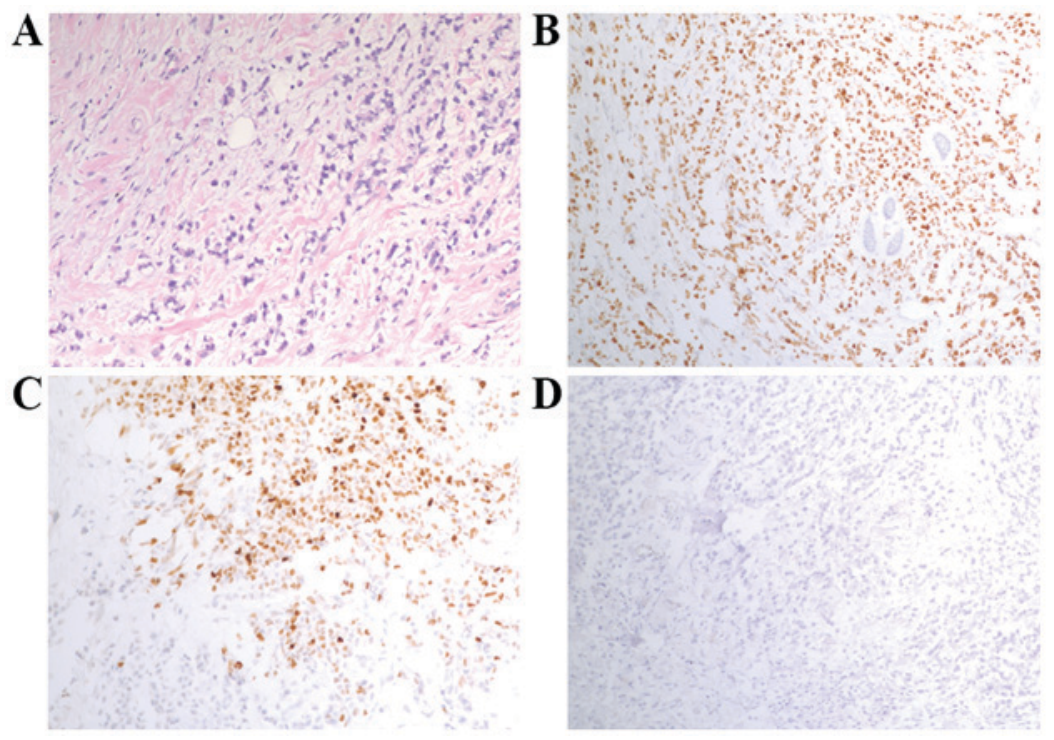

Figure 4. Pathology of invasive lobular carcinoma of the breast. (A) Hematoxylin and eosin staining. (B) ER, (C) PR and (D) HER2 staining. ER and PR results were positive; however, HER2 results were negative. Magnification, x200. HER2, human epidermal growth factor receptor 2; ER, estrogen receptor; PR, progesterone receptor.
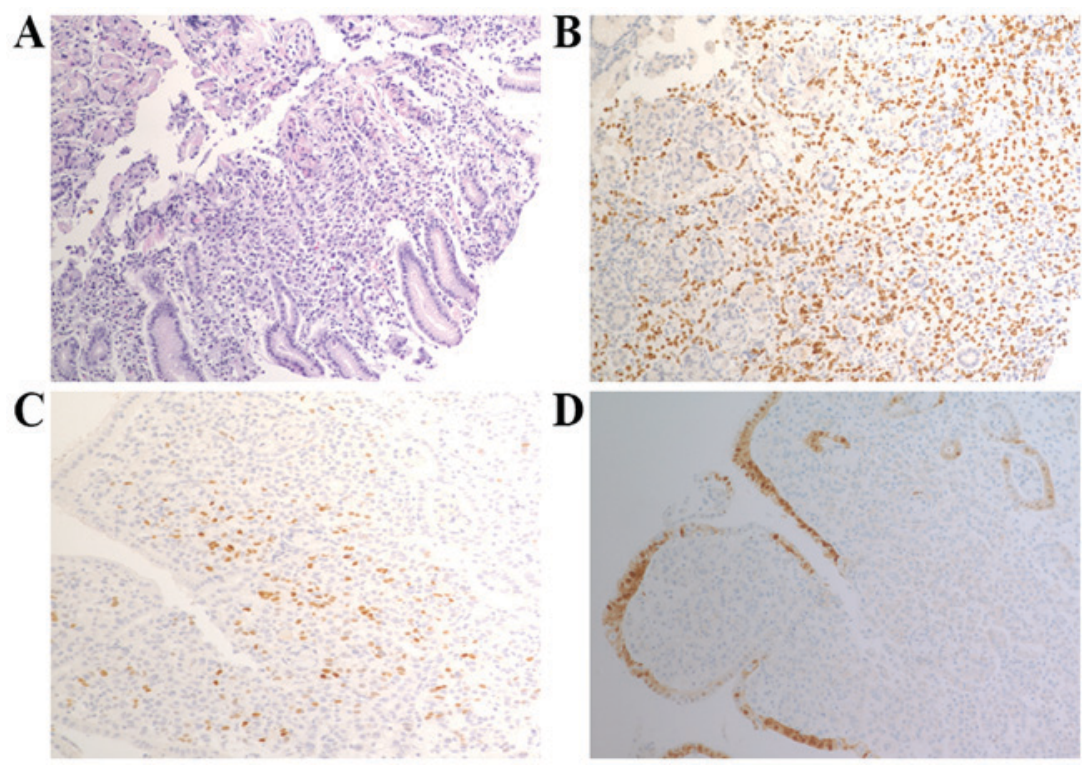

Figure 5. Pathology of gastric metastasis. (A) Hematoxylin and eosin staining. (B) ER, (C) PR and (D) HER2 staining. ER and PR results were positive; however, HER2 results were negative. Magnification, x200. HER2, human epidermal growth factor receptor 2; ER, estrogen receptor; PR, progesterone receptor.

\section{Discussion}

Breast cancer is a heterogeneous disease. In the present study, it was not possible to precisely discern which type of breast cancer metastasized most often to the stomach. However, luminal type A or B breast cancer tended to commonly metastasize to the stomach. Furthermore, ILC metastasis to the stomach was more frequent when considering pathological type.

There are several case reports including only a few cases due to low incidence of breast cancer with gastric metastasis (6-16). Taal et al (21) reported 51 cases of breast cancer with gastric metastasis, and 36 of the 51 patients had ILC. In contrast with a favorable outcome in the current study, median survival was 10 months and the 2-year survival rate was $23 \%$ in Taal et al study. This may be due to advancements in breast cancer treatment as enrollment of patients was between 1977 and 1997, and cancer treatment was administered prior to 2000. Xu et al (22) also reported 78 cases of metastatic gastric cancer from breast carcinoma from a literature search, which included Taal et al study. Of the 78 cases, 51 had ILC and positive IHC staining for ER, PR and HER-2 was $94.0,68.3$ and $5.9 \%$, respectively. This result is very similar to the current study, in which positive IHC staining for ER, PR and HER-2 was 81.8, 50, and 0\%, respectively. However, in contrast with $\mathrm{Xu}$ et al's study, which comprised a broad spectrum of patients regarding study time and medical 


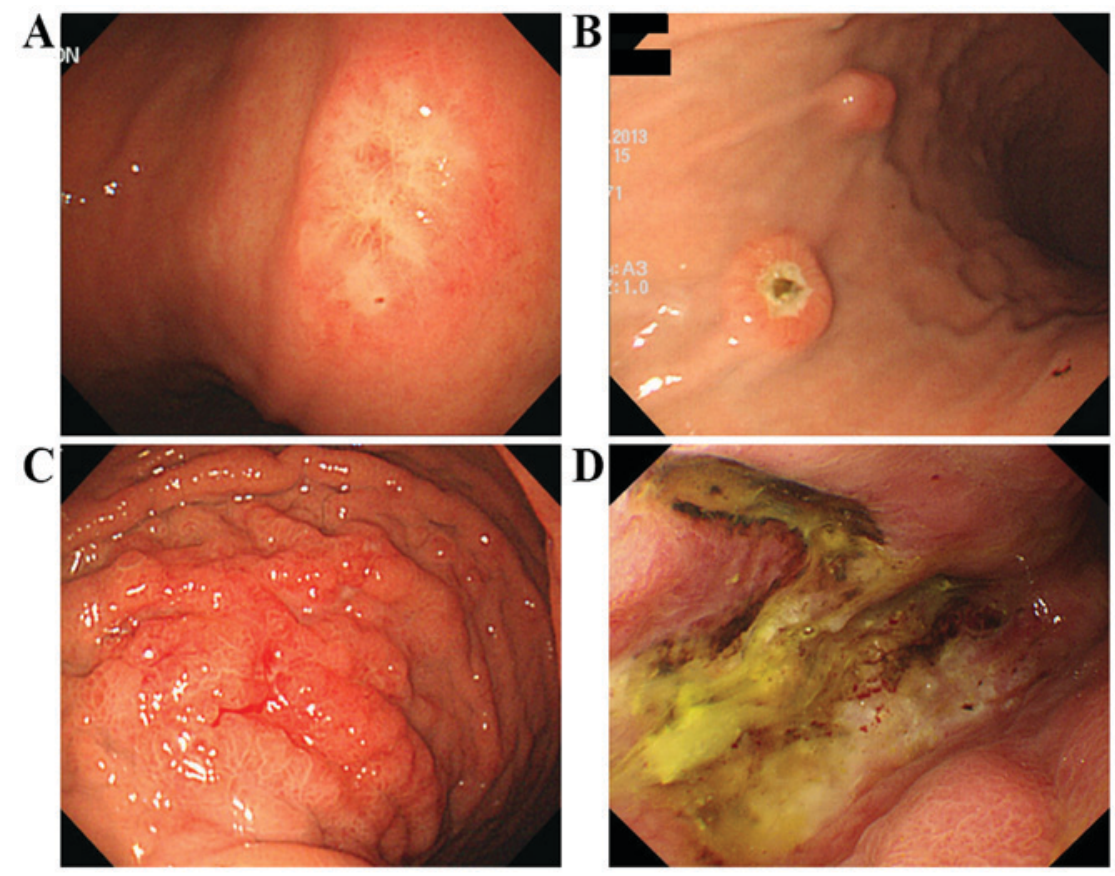

Figure 6. Endoscopic results of gastric metastasis. (A) White discolored mucosal lesion with speculated edge. (B) Round polypoid lesions with or without ulceration. (C) Diffuse infiltrative lesion with spontaneous bleeding and fold thickening mimicking Bormann type IV. (D) Deep ulcerative lesion with yellowish exudate and spontaneous bleeding.

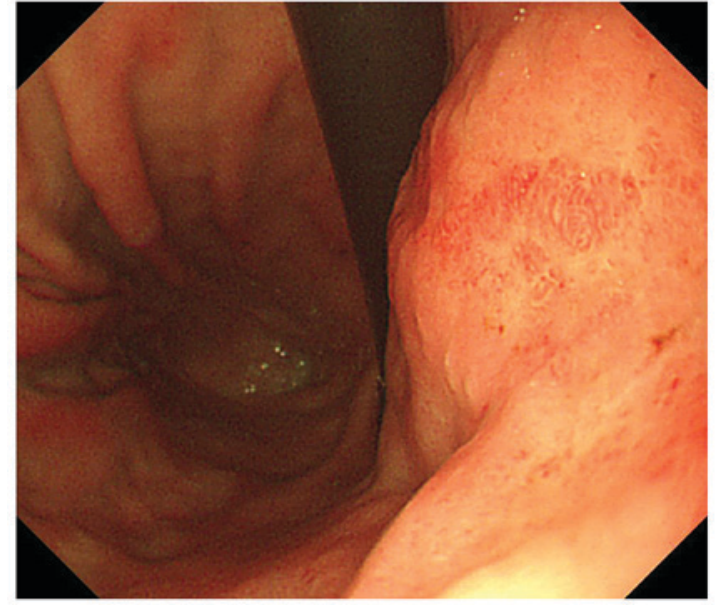

Figure 7. Diffuse infiltrative lesion with hyperemic mucosal change in the remnant stomach following a Whipple procedure.

centers, the current study only collected data from a single center and in a shorter time period.

Among the 66 patients diagnosed with gastric carcinoma by biopsy pathology, 47 had double primary cancer based on clinical situation or pathology reports. Out of the 47 patients with double primary cancer, 38 had no evidence of breast or gastric cancer recurrence according to their follow-up. Among these 38 patients, 34 underwent gastrectomy and 4 underwent endoscopic resection. Certain patients who had double primary cancer may have had breast cancer with gastric metastasis, which may have not been identified due to lack of attention to patient history regarding breast cancer at the time of diagnosis. Therefore, only 2 out of 47 patients with double primary cancer had stomach samples with ER/PR
IHC staining, and 3 had stomach samples with GCDFP-15 IHC staining. These results were in contrast with those of the 13 patients with breast cancer and gastric metastasis, 11 of whom had IHC staining results for ER, 10 for PR, 9 for HER2 and 10 for GCDFP-15. HER2 IHC staining was performed for samples from 37 patients, as HER2 is a routine IHC examination for gastric cancer. Nevertheless, 38 out of 47 patients had no evidence of recurrence. Even if recurrence following gastrectomy occurred, pathological stage was higher than stage III. In summary, a number of indications may be considered at the time of IHC staining, particularly the results of ER/PR and GCDFP-15 IHC staining. The stomach was the first metastatic lesion location in $6(54.5 \%)$ out of 11 patients with breast cancer de novo stage I-III cancer, and the median time interval from breast cancer diagnosis to gastric metastasis was 77.5 months. This interval of $>5$ years may be long enough to consider a gastric malignancy as a primary cancer. The results of the present study suggests that clinical suspicion and thorough pathological review are required.

The 3-year survival rate following gastric metastasis was $79.1 \%$ and the estimated mean survival time was 35.1 months. The 13 patients with breast cancer with gastric metastases had a more favorable outcome compared with patients with gastric cancer stage IV (23). A total of 2 patients succumbed, emphasizing that classifying gastric malignancy as primary or secondary is important for deciding adequate treatment without unnecessary surgery in addition to estimating prognosis.

Limitations of the present study included that it was small, retrospective and based in a single center. Due to these limitations, it was not possible to demonstrate the associations among markers associated with breast cancer, including receptor status, tumor grade and survival. Additional cases are required to strengthen the present study. Secondly, information was incomplete. For example, certain IHC staining results 
were missing. With cautious suspicion, the number of cases of breast cancer with gastric metastasis may increase, and additional information may be gathered. The number of patients with double primary cancer may have been underestimated in the present study. As gastric cancer was the variable searched for in pathology reports, data on gastric lymphoma, gastrointestinal stromal tumors, gastric neuroendocrine tumors and other types of carcinoma may have been missed. However, as the proportion of the aforementioned types of cancer was low and the aim of the present study was to analyze breast cancer with gastric metastasis, patients may not have been missed from the analysis process after all. Further translational research for gastric metastases from breast cancer to elucidate the underlying biological mechanism is required.

The present study concluded that breast cancer with gastric metastasis is rare. Luminal-type breast cancer or ILC tended to metastasize to the stomach more than other types of breast cancer. The stomach was one of the first metastatic lesion locations among patients with breast cancer and gastric metastasis, and these patients had a more favorable prognosis compared with patients with primary gastric cancer. Since gastric metastasis from breast cancer is rare, a thorough pathology review and greater clinical suspicion are required.

\section{Acknowledgements}

Not applicable.

\section{Funding}

No funding was received.

\section{Availability of data and materials}

The datasets used and/or analyzed during the current study are available from the corresponding author on reasonable request.

\section{Authors' contributions}

$\mathrm{JH}$ gathered, analyzed and interpreted the patient data and wrote the article. YK, JC, SWL, SEP, HKK and HL gathered and analyzed the patient data. SYC analyzed and interpreted the pathology of the patients. JK, JSA, YHI and YHP analyzed and interpreted the patient data. All authors read and approved the final manuscript.

\section{Ethics approval and consent to participate}

This study was approved by the Institutional Review Board of Samsung Medical Center (approval no. 2017-11-097-002), and the requirement for informed consent was waived.

\section{Patient consent for publication}

Not applicable.

\section{Competing interests}

The authors declare that they have no competing interests.

\section{References}

1. Siegel RL, Miller KD and Jemal A: Cancer statistics, 2016. CA Cancer J Clin 66: 7-30, 2016.

2. Foulkes WD, Smith IE and Reis-Filho JS: Triple-negative breast cancer. N Engl J Med 363: 1938-1948, 2010.

3. Harris M, Howell A, Chrissohou M, Swindell RI, Hudson M and Sellwood RA: A comparison of the metastatic pattern of infiltrating lobular carcinoma and infiltrating duct carcinoma of the breast. Br J Cancer 50: 23, 1984.

4. Kennecke H, Yerushalmi R, Woods R, Cheang MCU, Voduc D, Speers CH, Nielsen TO and Gelmon K: Metastatic behavior of breast cancer subtypes. J Clin Oncol 28: 3271-3277, 2010.

5. Patanaphan V, Salazar OM and Risco R: Breast cancer: Metastatic patterns and their prognosis. South Med J 81: 1109-1112, 1988.

6. Abe T, Yoshidome K, Iijima H, Oyama T, Akamatsu H, Kawai N, Yasumaru M, Tsujimoto M, Nishida T and Tsujii M: Metastatic breast cancer mimicking primary depressed gastric cancer. Gastroint Endosc 70: 1241-1242, 2009.

7. Arrangoiz R, Papavasiliou P, Dushkin H and Farma JM: Case report and literature review: Metastatic lobular carcinoma of the breast an unusual presentation. Int J Case Rep 2: 301-305, 2011.

8. Aurello P, D'Angelo F, Cosenza G, Petrocca S, Stoppacciaro A, Ramacciato G and Ziparo V: Gastric metastasis 14 years after mastectomy for breast lobular carcinoma: Case Report and Literature Review. Am Surg 72: 456-460, 2006.

9. Brandi G, Campadelli E, Nobili E and Leone O: Breast carcinoma presenting as linitis plastica. Dig Liver Dis 42: 306, 2010.

10. Dumoulin FL and Gupta RS: Breast cancer metastasis to the stomach resembling small benign gastric polyps. Gastrointest Endosc 69: 174-175, 2009

11. Ellis MC, Mason T, Barnett J, Kiesow LL and Vetto JT: Gastric malignancies in breast cancer survivors: Pathology and outcomes. Am J Surg 197: 633-636, 2009.

12. Kudo T, Matsumoto T, Nakamura S, Nakamura S, Esaki M, Yada S, Hirahashi M, Yao T and Iida M: Solitary minute metastasis from breast cancer mimicking primary intramucosal gastric signet-cell cancer. Gastrointest Endosc 62: 139-140, 2005.

13. Pectasides D, Psyrri A, Pliarchopoulou K, Floros T, Papaxoinis G, Skondra M, Papatsibas G, Macheras A, Athanasas G, Arapantoni-Datioti P and Economopoulos T: Gastric metastases originating from breast cancer: Report of 8 cases and review of the literature. Anticancer Res 29: 4759-4763, 2009.

14. Rusticeanu M, Schuster M, Moga SL, Solomayer E-F, Bohle RM, Lammert F and Zimmer V: Metastatic lobular breast cancer presenting as gastric linitis plastica. Am J Med 124: e5-e6, 2011.

15. Schwarz RE, Klimstra DS and Turnbull ADM: Metastatic breast cancer masquerading as gastrointestinal primary. Am J Gastroenterol 93: 111-114, 1998.

16. Whitty LA, Crawford DL, Woodland JH, Patel JC, Nattier B and Thomas CR: Metastatic breast cancer presenting as linitis plastica of the stomach. Gastric Cancer 8: 193-197, 2005.

17. Müller A, Homey B, Soto H, Ge N, Catron D, Buchanan ME, McClanahan T, Murphy E, Yuan W, Wagner SN, et al: Involvement of chemokine receptors in breast cancer metastasis. Nature 410: 50-60, 2001.

18. Edge SB and Compton CC: The American Joint Committee on Cancer: The 7th edition of the AJCC cancer staging manual and the future of TNM. Ann Surg Oncol 17: 1471-1474, 2010.

19. Laurén P: The two histological main types of gastric carcinoma: Diffuse and so-called intestinal-type carcinoma. Acta Pathol Microbiol Scand 64: 31-49, 1965.

20. Hicks DG and Schiffhauer L: Standardized assessment of the HER2 status in breast cancer by immunohistochemistry. Lab Med 42: 459-467, 2011.

21. Taal BG, Peterse H and Boot H: Clinical presentation, endoscopic features, and treatment of gastric metastases from breast carcinoma. Cancer 89: 2214-2221, 2000.

22. Xu L, Liang S, Yan N, Zhang L, Gu H, Fei X, Xu Y and Zhang F: Metastatic gastric cancer from breast carcinoma: A report of 78 cases. Oncol Lett 14: 4069-4077, 2017.

23. Orditura M, Galizia G, Sforza V, Gambardella V, Fabozzi A, Laterza MM, Andreozzi F, Ventriglia J, Savastano B, Mabilia A, Lieto E, et al: Treatment of gastric cancer. World J Gastroenterol 20: 1635-1649, 2014. 